\title{
The dark side of visual awareness in sport: Inattentional blindness in a real-world basketball task
}

\author{
Philip Furley and Daniel Memmert \\ German Sport University Cologne, Cologne, Germany \\ AND \\ Christian Heller \\ University of Heidelberg, Heidelberg, Germany
}

\begin{abstract}
Most research in the field of decision making in sports has focused on the bright side of visual attention and has not taken the dark side of visual awareness into account. Understanding the costs of such inattention should be complementary to the study of how attention facilitates perception. In the present study, we provide evidence for the existence of inattentional blindness (IB) in a real-world basketball setting among adults (Experiment 1). In Experiment 2, we found that players with hardly any basketball experience were more likely to experience IB in a real-world basketball setting, as compared with experienced athletes. Improving the ecological validity of the setting by enhancing the perception-action coupling (Experiment 3) and increasing task difficulty (Experiment 4) did not appear to affect the occurrence of IB among experienced athletes. IB can be considered a limitation of the visual system, but it also highlights a critical aspect of visual processing, which allows us to remain focused on the important aspects of the world. But as is shown in the present experiments, it is possible to induce an attentional set - for example, by sport-specific instructions - that leads to players' missing important game-relevant information.
\end{abstract}

Since the performance of many sports, especially team ball games like basketball, soccer, water polo, field and ice hockey, and football, frequently requires concurrent performance of two or more skills (e.g., carrying the ball while visually scanning for teammates to pass to), understanding more about attention processes is essential to understanding performance in sports. Most research on decision making in sports has focused on the bright side of visual attention and has not taken the dark side of visual awareness, or inattention, into account (for an overview, see Abernethy, Maxwell, Masters, van der Kamp, \& Jackson, 2007). This is unfortunate, since Abernethy (2001) referred to selective attention as a double-edged phenomenon in team ball sports; it is both a blessing, in terms of helping the player to overcome potential distractions, and a curse, in situations in which attention needs to be simultaneously divided. If attention is too easily disrupted, athletes might have difficulty completing their task goals.

On the other hand, however, if selective attention is too effective, important events might fail to reach consciousness. Understanding the costs of such inattention should be complementary to the study of how attention facilitates perception (Chun \& Marois, 2002), also in the field of sport. Extreme selectivity does produce greater focus and efficiency but also can result in inattentional blindness (IB) for unexpected events (e.g., Mack \& Rock, 1998; Simons \& Chabris, 1999). The term inattentional blindness refers to the failure to detect an unexpected object or event if attention is diverted to another task or object, even if it is right in front of the observer. IB has been shown to be a highly robust finding in the field of psychology (Mack \& Rock, 1998; Most, Scholl, Clifford, \& Simons, 2005; Simons \& Chabris, 1999), showing that participants tend to miss an unexpected event if their attention is engaged in some kind of attention-demanding task. Once participants start expecting unusual events, the latter become readily detectable. Therefore, this paradigm seems highly suitable and applicable for studying attention processes in the field of sports decision making - for example, to investigate the attentional benefits and costs of instructions from coaches or predetermined offensive plays in team sports.

It has long been known that humans have a limited information-processing capacity (for a review, see Broadbent, 1958; for a recent review, see Knudsen, 2007), and given the enormous amount of information that bombards players of team ball games, it becomes essential for performance efficiency that the most task-relevant (or pertinent)

P. Furley, p.furley@dshs-koeln.de 
information should actually get processed preferentially. Therefore, it is often not possible for a player to consider all the possibilities in complex situations, which means that he or she might consider only the most likely ones or the ones that his or her attention has been deliberately directed to by the instructions of a coach, such as in specific offensive game strategies. In this context, Williams, Davids, and Williams (1999) mentioned that "the coach should help performers to develop 'mind-sets' or expectations regarding which cues to attend to and which ones to ignore" (p. 54). Coaches of team ball sports frequently utilize instructions in order to overcome the limited processing capacities of their players by directing their focus of attention to what are considered information-rich areas. These attention-guiding instructions can potentially induce an attentional set (Most et al., 2005), directing the attentional focus of the players. This might be an explanation for those cases, observed daily, in which players who become suddenly free but are not part of a specific offensive play are often not perceived and, therefore, not passed to (Memmert, Simons, \& Grimme, 2009).

Interestingly, Memmert and Furley (2007) found preliminary evidence that IB exists among adolescents in team ball sports by using a laboratory handball-specific tactical-decision-making task in which participants were instructed to focus their attention on their direct opponent. In their study, participants viewed videos in a dual-task situation. The attention-demanding task was to name the position of their direct opponent while having to make a tactical decision that would most likely result in a goal (performance task). This is the standard procedure in realworld IB tasks such as that in Haines (1991), in which experienced pilots had to land a plane (performance task) while monitoring a heads-up display (attentiondemanding task). Other examples of real-world IB tasks have come from the area of driving (Most \& Astur, 2007; Strayer, Drews, \& Johnston, 2003). These real-world IB tasks showed evidence that performance in the activity of interest (e.g., driving or landing an airplane) of highly experienced adults significantly declined if their attention was drawn to another attention-demanding task, such as monitoring a heads-up display or talking on a cell phone. In accord with these findings, Memmert and Furley found that performance in tactical decision making declined among adolescent handball players when their attention was engaged in an attention-demanding monitoring task. Thus, it is important to understand more about the cost of such inattention in the field of sports.

Memmert and Furley (2007) illustrated neatly that instructions intended to facilitate perception can, in fact, be detrimental for the decision-making process by reducing the attentional flexibility of adolescent athletes. Flexibility of attention is usually defined as the ability to quickly disengage, move, and engage attention on various locations in space (Tenenbaum \& Bar-Eli, 1995). Various studies using the Posner (1980) cuing paradigm were able to show an attentional benefit for cued or expected locations and an attentional cost for uncued or unexpected locations. Meanwhile, numerous studies using sport-specific stimuli have also utilized the cuing paradigm (e.g., Castiello
\& Umiltà, 1992; Enns \& Richards, 1997; Lum, Enns, \& Pratt, 2002; Pesce-Anzeneder \& Bösel, 1998). Taking this into account, one might plausibly argue that instructions from coaches could have a similar effect, leading to an attentional benefit of expected game situations and an attentional cost of unexpected situations, since Nougier and Rossi (1999) plausibly stated that the subjective or objective knowledge of the respective probabilities for different events is typically the basis of a voluntary orienting of attention. Thus, one may conclude that, the higher the probability - for example, derived from instructions of a coach - of a specific event is, the greater the attention paid to it will be. Interestingly, the findings from the attentional-orienting literature in sports suggest otherwise by indicating that expert athletes seem to prefer to pay proportionally less attention to highly likely events and more attention to less likely events (for reviews, see Memmert, 2009; Nougier \& Rossi, 1999).

A plausible explanation for this might be that athletes participating in sports with high situation uncertainty, such as handball, basketball, or soccer, benefit from minimizing the time needed to shift visual attention toward relevant but unexpected information. Therefore, various researchers in the field of attentional orienting in sports (Cereatti, Casella, Manganelli, \& Pesce, 2009; Nougier \& Rossi, 1999) have claimed that experienced fast ball game players adopt an expecting-the-unexpected strategy. This strategy gives experienced athletes the advantage of rapidly zooming out visuospatial attention, allowing them to process unexpected but useful information (Pesce, Tessitore, Casella, Pirritano, \& Capranica, 2007). Taking this into consideration, one might expect experienced athletes to be less prone to the occurrence of IB in a real-world IB task. This assumption is in line with the results in Memmert (2006), who found that children showed significantly more IB than did adults in Simons and Chabris's (1999) IB task. In regard to the attentional-orienting literature, one might expect experienced athletes to show even lower levels of IB in a real-world IB task.

Another line of argumentation as to why experienced adults might be less prone to IB in a task closely related to their domain of expertise stems from the sport expertise literature (Ericsson, Krampe, \& Tesch-Römer, 1993; Williams \& Ericsson, 2005; for a recent review, see Williams $\&$ Ford, 2008). According to this, one might argue that, since the test material utilized by Memmert and Furley (2007) was designed for adolescents (simple game situation, in which the last frame was visible for $3 \mathrm{sec}$ ), it did not demand enough attentional resources from adults, since Williams and Ford noted that increased experience of game situations leads to perceptual-cognitive adaptations in team ball players. These perceptual-cognitive advantages in team ball sports are most likely due to the following: pattern recognition (Tenenbaum, Levy-Kolker, Sade, Liebermann, \& Lidor, 1996; Williams, Hodges, North, \& Barton, 2006), which is the ability to detect meaningful patterns of play early in their development; superior visual search behaviors, such as fixation duration, number of fixations, and the proportion of time spent fixating specific areas of the display (for a review, see Vaeyens, Lenoir, Williams, 
Mazyn, \& Philippaerts, 2007); and the ability to accurately estimate situational probabilities, allowing them to free attentional resources that can be allocated to the most important contextual cues (Ward \& Williams, 2003). Therefore, adults should have an advantage over adolescents in tasks such as that in Memmert and Furley via the described abilities, allowing them to free attentional resources that can potentially be engaged in the tactical-decision-making process. These findings seem to indicate that adults, with year-long experience in their sports, should be less prone than adolescents to showing IB in a similar task. This is in line with the prediction from the attentional-orienting literature suggesting that expert athletes might prefer to pay proportionally more attention to less likely events than to highly likely events (Nougier \& Rossi, 1999).

Therefore, IB might not occur to the same degree among experienced adult athletes, since the efficiency of orienting of attention increases with age (Brodeur \& Enns, 1997; Nougier, Azemar, Stein, \& Ripoll, 1992), and because of the described perceptual-cognitive adaptations that occur among expert athletes (Williams \& Ford, 2008). For these reasons, we hypothesized that, in a similar realworld IB task, IB would occur to a lesser degree among adults than was evident for the adolescent participants in Memmert and Furley (2007). This issue was addressed in Experiment 1.

\section{EXPERIMENT 1}

\section{Method}

Participants. Twenty male basketball players $(M=23.7$ years, $S D=3.0$ ) participated in Experiment 1. They had been playing basketball for an average total of 9.3 years $(S D=3.8)$ continuously at a level not below the fifth highest league in Europe. Each participant volunteered and did not receive any kind of compensation for participation. Informed consent was obtained before commencing the experiment.

Stimuli. The stimulus materials were video sequences of a fiveon-five basketball game situation involving two adult teams. The development of the basketball IB task (BIBT) involved making 40 video sequences in which five attackers wearing green basketball jerseys were playing five defenders wearing white jerseys, just as in a real basketball game. The camera was positioned at the same level as the players and captured the entire half court in which the action took place. The crucial player in the BIBT was one of the green offensive players, who was marked with a bright red circle around his body at the beginning of each video. This player always got the ball right at the end of the video. The starting formation of both the offensive and the defensive teams was the same for all sequences. Whereas the defensive team was instructed to play a man-to-man defense strategy, the offensive play developed from a one-two-two strategy, with one point guard, two forwards, and two center players occupying the low post position (see Figure 1, top panel). Both the offensive and the defensive teams had received precise instructions on how to move and pass the ball, resulting in an intended final frame. In this final frame, the attacker, marked red at the beginning, always received the ball before the video came to an end. Furthermore, the direct opponent of this player was instructed to occupy either a position very close to his opponent (less than $2 \mathrm{ft}$ away from the attacker) or a position far away from his opponent (at least $3 \mathrm{ft}$ away from the attacker) in this final frame.

The video clips were selected through expert ratings and item analysis. Three experts, all of whom were in possession of the second highest basketball coaching license in Europe, rated the videos.
From the 40 sequences filmed, the test clips that were most authentic and most clearly constituted an obvious tactical solution were chosen. The experts were asked to find the best tactical solution for every video. In a second step, they rated every sequence according to the degree of obviousness of the chosen tactical decision, on a scale from 1 (totally obvious) to 5 (not at all obvious). On the basis of these expert ratings, we selected 4 sequences for the final version of the BIBT: 3 noncritical sequences and 1 critical sequence, which are specified below.

Noncritical trial. The first three trials were noncritical trials in which the video did not include an obviously unguarded player, with the first one being a practice trial in order to clarify the procedure for the participants.

Critical trial. On the fourth, critical trial - in contrast to the noncritical trials - there was an obviously unguarded player who would be the best player to pass to if the participant perceived him, as was shown by the expert ratings. In addition to the expert ratings, we further selected the clip in which the free player was closest to the defending player, on whom the participants had to focus their attention; this clip had been rated with one accord as involving a totally obvious decision (see Figure 1). This ensures that the free player was fully visible and close to the focus of attention, since this was directed to the defensive player. If the participant correctly identified the position of the white defensive player, which indicated that he had focused his attention on the defensive player, and also made the tactical decision to pass to the unguarded player, the experiment came to an end. If, however, the participant did not pass to the free player after the final frame, he was asked to continue with Trial 5 (the full-attention trial). This is the standard procedure in the IB paradigm.

Although it is problematic from an experimental perspective to have only one critical trial in order to determine whether participants detect an unexpected event, there is unfortunately no way around this procedure. Once one has shown an unexpected event, people will not treat future events as unexpected. Nevertheless, it is highly important to study IB - or more generally, the detection of unexpected events - in real-world settings. Rare unexpected events are, for example, a common cause of driving accidents and other mishaps - for example, in sport settings - and the only way to study the consequences of unexpected events and differences in their detectability is to make sure that they are actually unexpected. The only way to do that is to have just one critical trial.

Full-attention trial. On this last trial, the participants were told that, this time, they only had to make a tactical decision, without naming the position of their direct opponent; that is, they did not have to perform the attention-demanding task. What they did not know was that they were viewing exactly the same video as on the trial before. If the participants passed the ball to the free player in this full-attention trial and had not in the previous critical trial, the difference in performance could be attributed solely to the absence of the attention-demanding task (present in the critical trial, but not in the full-attention trial), and not to expertise-related effects.

Procedure. The BIBT was projected onto a large screen (horizontal $\times$ vertical: $3.2 \times 2.4 \mathrm{~m}$ ). The participants stood in front of the screen at a distance of $6 \mathrm{~m}$. The size of the stimulus field was $21.8^{\circ}$ of visual angle in the vertical dimension and $28.1^{\circ}$ of visual angle in the horizontal dimension. The experimenter asked the observers to read the instructions displayed on the screen and to indicate whether they had finished reading and understood the instructions. The participants were asked to take over the role of the green attacker marked red at the beginning of the video and to make a tactical decision for this player at the end of the video that would most likely result in a goal for their team by saying out loud, for example, "pass to left wing player," "cut," or "shoot." The instructions explicitly pointed out that all decision options were available to the participants.

On all five of the video trials, the first frame of the video appeared frozen for $3 \mathrm{sec}$ so that the observers had some time to orient themselves. The duration of the video sequences was about $15 \mathrm{sec}$. At the end of the video, the final frame froze and immediately switched to a white screen. Two tasks were assigned to all the participants 
A

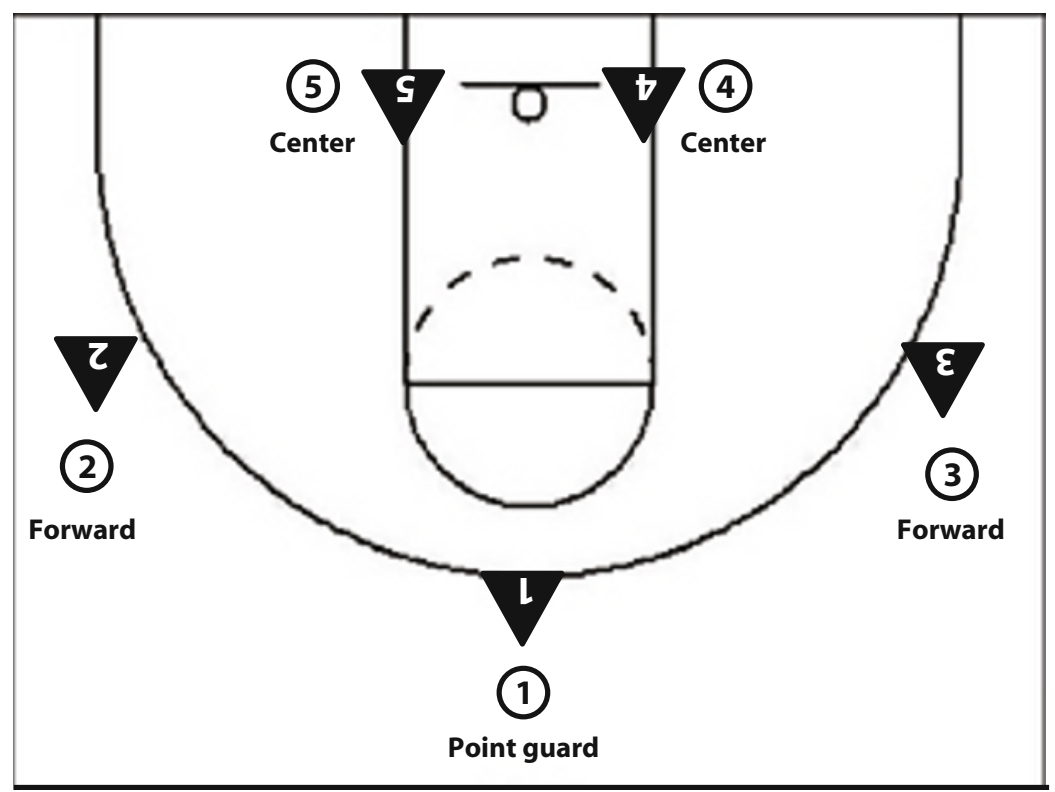

B

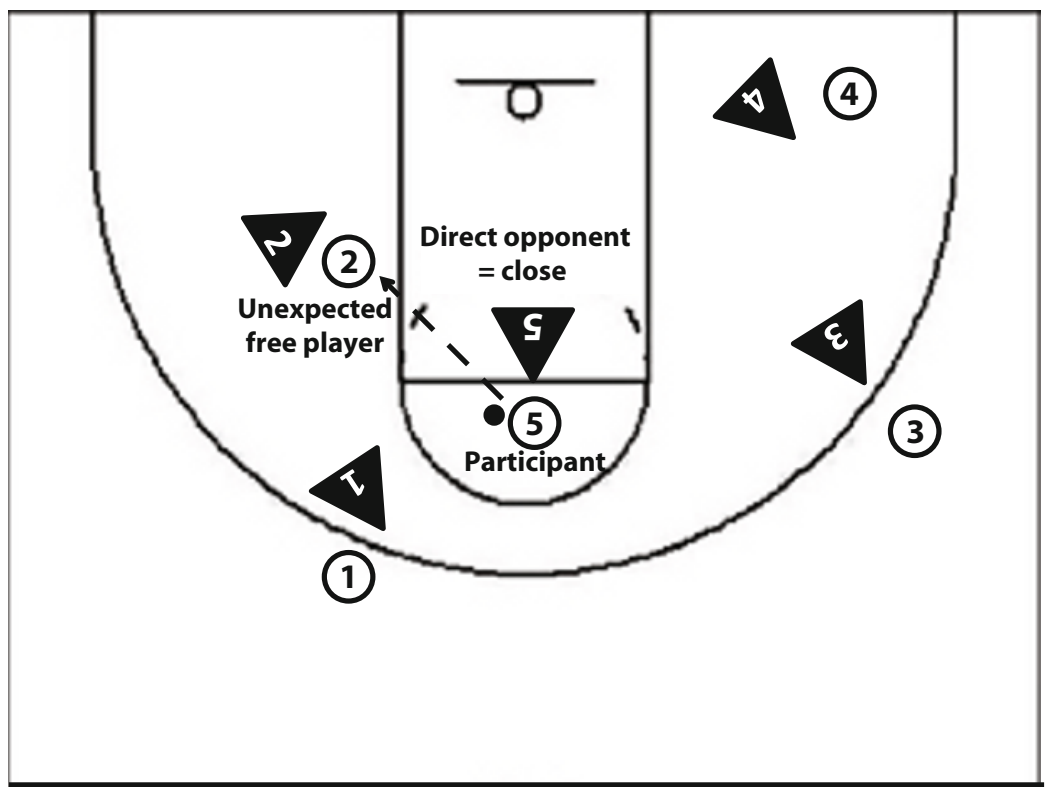

Figure 1. (A) Schematic drawing of the starting formation of both the offensive and the defensive teams. The offensive team adopted a one-two-two strategy, with one point guard, two forwards, and two center players occupying the low post position. The defensive team was instructed to play a man-to-man defense. (B) Schematic drawing of the last frame of the critical trial. The offensive team is represented by the white circles, with Player 5 in possession of the ball, for whom the participants had to make a tactical decision. The direct opponent, black Player 5, is occupying a position close to white Player 5, which had to be recognized in the attention-demanding task. The optimal solution is a pass to white Player 2, who is cutting to the basket, as indicated by the dotted arrow. 
for each of the 5 trials making up Experiment 1. For each trial, the participants viewed a short video as described above. At the end of each video, the participants had to pay attention to the position of their direct opponent and identify their white defender as being either close to them (i.e., less than $2 \mathrm{ft}$ away) or far away from them (i.e., more than $3 \mathrm{ft}$ away). In the second task, the participants were asked to make a tactical decision on the basis of the situation and position of the white defensive player identified in the first task. All the participants were told that they had to make a tactical decision that would most likely lead to a goal for their team and that identifying the position of the white defensive player might help them make their decision. The rationale for choosing a functional sport-specific attention-demanding task was to improve the ecological validity of the IB paradigm, since IB has been shown to be a very robust finding in the laboratory but only a few studies have investigated it in ecologically valid settings (for exceptions, see Haines, 1991; Most \& Astur, 2007; Strayer et al., 2003), and thereby emphasize the practical relevance of IB in the field of team ball games. The position of the direct opponent in basketball can have a major impact on the tacticaldecision-making process; for example, if the defensive player is far away, a jump shot might be a good tactical decision, whereas cutting to the hoop might be a good decision if the direct opponent is close. After the video came to an end, the projected picture switched to a completely white screen, and the participants called out either "near" or "far" (attention-demanding task) and named the tactical decision that they would carry out (performance task).

After completing the trials, the participants answered follow-up questions designed to gather demographic information, determine whether they had been familiar with this or other related experiments prior to participation, and find out whether they had noticed that they had been watching the same video on the critical trial and the full-attention trial.

\section{Results and Discussion}

None of the participants claimed to know of the phenomenon and/or experimental paradigm of IB. Of the 20 participants, 4 were excluded from the final data analysis for not having passed to the open player in the full-attention trial, as was done in Memmert and Furley (2007). This was probably due to the highly complex game situation and the final frame's disappearing without any delay, which was a necessary modification of the IB task utilized by Memmert and Furley in order to investigate the occurrence of IB among adults, since the task would not have been challenging enough for adults. Furthermore, this is a general and well-known problem in the IB literature, in which approximately $15 \%$ to almost $30 \%$ of participants are excluded, due to the fact that there is only one critical trial (Mack \& Rock, 1998; Most et al., 2005; Simons \& Chabris, 1999).

All the participants solved the attention-demanding task correctly, which involved identifying the defensive player as close to them - that is, less than $2 \mathrm{ft}$ away. Of the 16 valid participants, 10 perceived the open player, and 6 failed to notice him (38\%). Thus, our results were slightly lower than the handball findings in Memmert and Furley (2007; 44\% failed to notice the unguarded player) and were comparable to the IB results in the psychology literature (Memmert, 2006; Most et al., 2005; Simons \& Chabris, 1999). Since the participants viewed exactly the same video on the critical trial as on the full-attention trial, the failure to pass to the open team member by $38 \%$ of the participants on the critical trial, even though they passed to him on the full-attention trial, can be attributed to their attention being engaged in the attention-demanding task (present in the critical trial and not in the full-attention trial) and, consequently, not expecting the suddenly open player. None of the participants experiencing IB reported that they had noticed anything unusual while performing the task. This indicates that the failure to pass to the open teammate resulted from IB, and probably not from the repetition of the video on the full-attention trial. Although this approach cannot fully eliminate the possibility that the participants noticed the unexpected event on the fullattention trial, because they were viewing the same video twice, the repetition explanation does not explain why the participants failed to notice the unexpected event on the critical trial, which, we argue, was due to the fact that attention was diverted to monitoring the opponent player. Therefore, it seems reasonable to say that even the experienced athletes, with year-long experience in their sport, did not pass to an obviously unguarded teammate when their attention was engaged in an additional sport-specific attention-demanding task-identifying the position of their direct opponent - to whom they did pass if this task was not present. This result is surprising and seems to suggest that the failure to detect the unguarded teammate can be attributed to the fact that their attention was engaged in monitoring their direct opponent. Taking into consideration the literature we have described on the increased efficiency of orienting of attention among experienced athletes (Brodeur \& Enns, 1997; Nougier et al., 1992) and on the perceptual-cognitive adaptations occurring among expert athletes (Williams \& Ericsson, 2005; Williams \& Ford, 2008), the result pattern that we found could not have been expected. Thus, our finding gives further evidence that IB is a very robust phenomenon that needs to be taken seriously in the field of team sports.

\section{EXPERIMENT 2}

Although the finding that IB occurs among experienced athletes is interesting, comparing the present results with the results found by Memmert and Furley (2007) raises problems, given the changes made in the basketball IB task. For this reason, we incorporated an expert and a novice group into Experiment 2, in order to investigate whether IB would occur less among experts in a task related directly to their domain of expertise. A study by Memmert (2006) investigated whether the degree of basketball expertise would predict the occurrence of IB in Simons and Chabris's (1999) general IB task, which was only very loosely linked to the domain of expertise of basketball players, since it is not common to have to count the number of passes, or even less, to perceive a man in a gorilla costume. The results indicated that basketball experts showed lower levels of IB and, thus, perceived the unexpected object more frequently than did novices, indicating that experts were better able to perceive an unexpected object in a task that was only loosely related to their domain of expertise. The author argued that basketball experts have acquired special perceptual skills in their year-long practice that enable them to process stimuli that 
are less likely to occur in a given situation. For this reason, it seems plausible that basketball experts should show even lesser degrees of IB, as compared with novices, in the basketball-specific task.

Another issue in Experiment 1 was the number of participants who did not pass to the open player on the fullattention trial. Since 4 players did not pass to the open player on the full-attention trial, it seems feasible that the optimal tactical solution identified by the experts' ratings might not have been so obvious for all the participants. We addressed this question by showing the participants the final frame of the critical trial without a time limit after the experiment and asking them to name the best tactical solution for this situation and to rate the solution on a scale of 1 (not at all obvious) to 5 (totally obvious). Furthermore, if this rating differed from the tactical decision on the full-attention trial, the participants were asked whether they had been aware of the solution chosen in the rating during the full-attention trial. Beyond this, we asked the participants who did not pass to the open player on the critical trial and did on the full-attention trial whether they had been aware of the open player during the fullattention trial.

\section{Method}

Participants. Altogether, 34 people participated in Experiment 2. The novice group $(M=22.4$ years, $S D=1.4)$ included 18 students from the German Sport University Cologne who had taken a basic basketball class but had never played basketball competitively. In this way, we could ensure that the novice participants had enough basketball knowledge to complete the task but were distinguishable from the expert group by not having any competitive basketball experience. The expert group consisted of $16(M=27.7$ years, $S D=$ 3.5) competitive basketball players who had continuously been playing not below the level of the fourth highest league in Europe. They had been playing for an average of 11.2 years $(S D=2.4)$. None of the participants in Experiment 1 participated in Experiment 2. Each participant had volunteered and did not receive any kind of compensation for participation. Informed consent was obtained before commencing the experiment.

Stimuli and Procedure. All the observers were tested individually with the BIBT. The stimulus materials were identical to those in Experiment 1. The only differences from Experiment 1 were that all the participants were shown the final frame of the critical trial, including the unexpected open player, at the end of the experiment, had to name the optimal tactical solution for the scene, and had to rate it according to how obvious the solutions were. Another difference was that the participants who did not pass to the open player on the critical trial and did on the full-attention trial were asked whether they had been aware of the open player during the full-attention trial. Furthermore, since Memmert and Furley (2007) found no differences between presenting the stimulus materials on a computer monitor and presenting them on a large screen, the BIBT was presented on a 15-in. laptop monitor on this trial. The participants sat on a chair $45 \mathrm{~cm}$ away from the screen. The size of the stimulus field was $27.1^{\circ}$ of visual angle along the vertical dimension and $33.7^{\circ}$ of visual angle along the horizontal dimension.

\section{Results and Discussion}

None of the participants claimed to know of the phenomenon and/or experimental paradigm of IB. Of the 34 participants, 9 were excluded from the final analysis. In the novice group, 6 were excluded: 2 for not identifying the position of the opponent player correctly-indicating that they did not focus their attention adequately - and 4 for not passing the ball to the open player on the fullattention trial. In the expert group, 3 players were excluded: 2 for not having passed to the open player on the full-attention trial and 1 for not naming the position of the white defensive player correctly.

No differences in performance on the attentiondemanding task - naming the position of the white defensive player-were evident. Of the 12 valid novices, only 5 participants noticed the unexpected open player on the full-attention trial, whereas 7 participants failed to notice the unexpected open player (58\%). In the expert group, 9 participants noticed the unexpected open player, and 4 participants (31\%) failed to notice the unexpected open player. This result confirms our hypothesis that experienced basketball players are less prone to show IB in an IB task closely linked to their domain of expertise, as compared with basketball novices $\left[\chi^{2}(1, N=25)=\right.$ $4.280, p<.05]$. Comparing the results to those in Experiment 1 revealed no differences between the expert groups, with approximately $38 \%$ failing to perceive the unguarded player in Experiment 1 and $31 \%$ in Experiment $2\left(\chi^{2}<1\right.$, n.s.). The difference between the novice group in Experiment 2 and the expert group in Experiment 1 failed to reach significance $\left[\chi^{2}(1, N=28)=2.222, p=.136\right]$.

No differences were evident $\left(\chi^{2}<1\right.$, n.s. $)$ in the ratings for the final frame of the critical trial between novices and experts. The pass to the open team member was rated as the best solution by $89 \%$ of the novice group and $94 \%$ of the experts. Altogether, about $92 \%$ of the participants rated the open player as the optimal tactical solution for the situation. The expert group rated the open team member as slightly more obvious (3.94) than did the novice group (3.63). This finding shows that almost all the participants considered the unguarded player the best solution when given enough time to perceive him. Of the 6 participants failing to pass to the open player in the full-attention trial, only 2 participants (both novices) did not rate the pass to the open player as the optimal solution. The remaining 4 stated that they had not been aware of the open player on the full-attention trial. On the critical trial and the fullattention trial, participants have to view a highly dynamic game situation and make a split-second decision as soon as a particular player gets the ball. The fact that the players do not exactly know when they are going to have to make the decision and that the final frame disappears immediately makes the task of naming the optimal tactical decision highly demanding, similar to the demands of a reallife basketball game in which one suddenly receives the ball and is forced to make a split-second decision. Thus, it is not surprising that some participants do not make the optimal decision, even in the full-attention condition. Similarly, experienced players oftentimes make suboptimal decisions when they have to make a split-second decision in a real-world basketball game.

None of the players who passed to the open player on the full-attention trial and did not on the critical trial stated having been aware of the open player on the critical trial. This finding strengthens the claim that the failure to pass to the open player in the critical trial can be attributed to 
the attentional demands of identifying the position of the opponent player. Thus, the participants actually appeared to be blind to the openness of the player, instead of deciding not to pass to him.

Since both the experiments by Memmert and Furley (2007) and the present Experiments 1 and 2 were conducted in a laboratory setting with an emphasis on experimental control, it was necessary to show that IB also exists in ecologically more valid settings. Whereas Experiment 2 was conceptualized so as to address further methodological concerns besides investigating expert-novice differences within the IB paradigm, Experiment 3 was designed to strengthen the practical implications of the findings by strengthening ecological validity. In Experiment 3, we investigated whether actually carrying out the tactical decision via a motor response and, thus, creating a more dynamic and sport-related situation would influence the occurrence of IB, since the poor perception-action coupling in Experiments 1 and 2 might have influenced the result pattern (Ranganathan \& Carlton, 2007).

\section{EXPERIMENT 3}

The findings from Experiments 1 and 2 have very important practical implications for coaches when they give instructions and feedback to their athletes. To strengthen the finding of Experiments 1 and 2, it is essential to further improve the ecological validity of the real-world basketball task. A major limitation of a large body of research in sport psychology is that perception-action coupling is poor when participants have to respond to visual stimuli by using verbal, written, or buttonpressing responses instead of actual motor responses (Ranganathan \& Carlton, 2007). For this reason, laboratorybased testing procedures requiring non-sport-specific responses, such as pressing a button or naming the tactical decision, as utilized in the studies of Memmert and Furley (2007), have their limitations for studying attention in the field of sport. Research in the field of sport (Farrow \& Abernethey, 2003), examining the influence of coupled and uncoupled perception-action environments, showed that coupled responses were better suited for predicting the direction of tennis serves than were uncoupled responses. Thus, one might argue that the occurrence of IB among the experienced athletes was due to the artificial laboratory situation. Therefore, it was necessary to replicate the experiments with participants actually having to carry out their tactical decision in the form of a motor response. In order to replicate the present findings in ecologically valid circumstances, as defined by Davids, Button, Araújo, Renshaw, and Hristovski (2006), we created a laboratory setting in Experiment 3 in which participants had to carry out a sport-specific motor response after viewing the stimulus material. We hypothesized that no differences in the pattern of results would emerge, since the results of Experiments 1 and 2 showed that experienced athletes fail to detect an open teammate when their attention is diverted to a monitoring task; since the detection of an open team member is the prerequisite for passing to this player, having to actually carry out the motor response should not matter. But since presented IB findings for sport-related situations have such important practical implications, it was necessary to replicate the findings of Experiment 1 in highly ecologically valid settings.

\section{Method}

Participants. The participants were 19 male basketball players $(M=24.8$ years, $S D=3.0)$ who had practiced the sport for an average total of 8.3 years $(S D=2.8)$ continuously not below the level of the fifth highest league in Europe. None of the participants in Experiments 1 or 2 participated in Experiment 3. Each participant volunteered to participate and did not receive any kind of compensation. Informed consent was obtained before commencing the experiment.

Stimuli and Procedure. All the observers were tested individually with the BIBT. This time, the participants were holding a basketball while watching the BIBT, and a basketball basket was installed above the screen. The stimuli and the task were exactly the same as those in Experiment 1, the only difference being that the participants were instructed to perform a real-world motor response by throwing at the basket, cutting to the basket, or passing (against a padded wall) in the direction of an open team member immediately after the video came to an end.

\section{Results and Discussion}

Data from 5 observers were discounted. One was excluded for not having named the position of the white defender correctly on the critical trial, indicating he had not focused his attention adequately, which is an essential requirement for the paradigm. Four participants were discounted because they did not pass to the open player on the full-attention trial. Again, all of the now remaining participants solved the attention-demanding task correctly. Of the 14 valid participants, 5 (36\%) failed to pass the ball to the open team member on the critical trial, although they did so on the full-attention trial. No differences from Experiment 1 were evident $\left(\chi^{2}<1\right.$, n.s.). Thus, carrying out a motor response did not change the results. Although the ecological validity of the experimental setting was improved by increasing the perception-action coupling, the occurrence of IB remained unchanged. This finding strengthens our point that IB is an important phenomenon in team sports and needs to be taken seriously by coaches, athletes, and scientists in the domain of sports, since tactical instructions that are given with a helpful intent might have a negative effect for both adolescents and adults.

In order to further reinforce this point, we manipulated the difficulty of the attention-demanding task in Experiment 3, since Simons and Chabris (1999) showed that varying the difficulty of the attention-demanding task changes the occurrence of IB.

\section{EXPERIMENT 4}

In Experiment 4, we investigated the effect of manipulating the attention-demanding task. Simons and Chabris (1999) showed that increasing the task difficulty of the attention-demanding task also increased the occurrence of IB. Therefore, we hypothesized that increasing the demands of the attention-demanding task would require more 
attentional resources of the participants and, therefore, performance on the performance task would decline.

It is not common in adult basketball to consciously pay attention to the distance of the opposing player. An interview of basketball trainers and players indicated that the positioning of the feet of the direct opponent is highly important when determining one's tactical decision (e.g., faking in one direction and cutting to the opposite) and, therefore, also contributes to the ecological validity of the BIBT. So we introduced a three-alternative forced choice task (left foot front, right foot front, feet parallel), as opposed to a two-alternative forced choice task (close, far). We assumed that deciding between three alternatives would demand more attentional resources than deciding between two alternatives, thus resulting in higher levels of IB. Furthermore, it seems to be a lot harder to discriminate between the positioning of feet than to identify the opponent as either far away or close. The idea of manipulating the difficulty of the attention-demanding task was derived from Simons and Chabris's (1999) original basketball task, in which differences in the occurrence of IB between an easy condition (just counting the total number of passes) and a hard condition (counting the number of passes and the bounces) were found.

The experimental setting was the same as that in Experiment 3 and took place in the same laboratory, with a slight variation of the attention-demanding task. This time, the participants had to focus on the positioning of the feet of their direct opponent at the end of each video. We hypothesized that the occurrence of IB would increase with this manipulation, since the three-alternative forced choice task would demand more attentional resources and, thereby, leave fewer for the tactical decision.

\section{Method}

Participants. The participants were 13 male basketball players $(M=27.5$ years, $S D=5.7)$ who had practiced the sport for an average total of 10.6 years $(S D=4.6)$ continuously not below the level of the fifth highest league in Europe. None of the participants in Experiments 1, 2, and 3 participated in Experiment 4. Each participant volunteered to participate and did not receive any kind of compensation. Informed consent was obtained before commencing the experiment.

Stimuli and Procedure. The only difference from Experiment 3 was the variation of the attention-demanding task. The participants were instructed to focus on the positioning of the feet of their direct opponent before they carried out their motor response and to name them as being parallel, left foot in front, or right foot in front. This instruction was clarified by showing exemplary pictures of the possible feet positions.

\section{Results and Discussion}

Data from 5 observers were discounted: 2 of them named the positioning of the feet wrongly, indicating they had not focused their attention correctly; the remaining 3 did not pass to the open player on the full-attention trial. The fact that, this time, 2 participants did not identify the positioning of the feet correctly indicates - although very marginallythat the attention-demanding task was indeed more difficult than that in Experiments 1 (all the participants named the position of the defender correctly) and 2 (1 participant failed to name the position correctly). Of the 8 valid participants,
$3(38 \%)$ failed to pass the ball to the open team member, although they did so on the full-attention trial. Again, the results in Experiments $1(38 \%)$ and $3(36 \%)$ were replicated $\left(\chi^{2}<1\right.$, n.s.). The manipulation of the attention-demanding task did not prove to be more attention demanding and, therefore, did not increase IB.

The results revealed that changing the attentiondemanding task from two possible response options to three did not increase the occurrence of IB. This might have been due to the fact that this manipulation only increased the ecological validity of the attention-demanding task but was not more attention demanding for adult basketball players. One could even argue that adult basketball players are more used to paying attention to the positioning of feet since, as the interviews indicated, positioning of the feet of a direct opponent is highly important when one determines one's tactical decision and, therefore, demands fewer attentional capacities, thus freeing them for decision-making processes. These considerations could be the topic of future investigations.

\section{GENERAL DISCUSSION AND CONCLUSION}

In all four experiments, it was possible to provide evidence for the occurrence of IB among experienced adult basketball players and show that IB is a highly robust phenomenon also in the field of team ball sports. The results discussed here provide further evidence for the existence of IB in real-world situations - specifically, in a basketball setting among adults - and support the findings in Memmert and Furley (2007). Simons (2007) stated that all of the following criteria must hold in order to classify perceptual failures as IB, as opposed to a different type of failure of awareness: (1) Observers fail to notice a visual object or event; (2) the object or event is fully visible, and observers readily see it if they are looking for it; (3) the failure to detect the unexpected object or event results from engagement of attention on other aspects of the display and not from aspects of the visual stimulus itself; and (4) the object or event is unexpected. The first three points are clearly fulfilled in the present study. Admittedly, one might argue that Point 4 might not be fulfilled, since the ball-possessing player always has to search for the best solution in a given situation and, therefore, an open player is unlikely to be unexpected. On the other hand, coaches frequently give specific instructions and introduce predetermined offensive strategies (e.g., American football, basketball, or handball) in order to reduce the complexity of the game and give guidance to the decision maker by directing his or her attentional focus (cf. Williams et al., 1999). These offensive strategies usually include only a subset of the players, and the decision maker therefore has to choose from only a limited number of possibilities. For this reason, it is possible that a player who is not part of a specific offensive strategy can be considered unexpected and is not incorporated into the decision-making process. This is precisely what was modeled in this study. A lot of offensive strategies result in similar if-then situations, such as the following: If the defense responds in manner A, then 
do B; if they respond in manner C, then do D. Note that we did not give our participants precise if-then rules, but the attention-demanding task, combined with the hint that this task might aid their decision, even though they were told that they had all the decision options available (pass, cut, shoot, etc.), seemed to have automatically triggered one of the most common internalized if-then rules in basketball: If my direct opponent is far away from me, then I will shoot; or, if he is close to me, then I will do a quick first step and cut to the basket. Following this argumentation, we consider that all of the criteria above were fulfilled in the present experiments and, therefore, the results can be attributed to IB, instead of some other processes at work.

As was argued in the introduction, IB can be considered a limitation of the visual system, but it also highlights a critical aspect of visual processing, which allows us to remain focused on the important aspects of the world or the sport game we are concurrently involved in. But as was shown in the experiments above, it is possible to induce an attentional set (Most et al., 2005) - for example, by specific instructions that lead to missing important gamerelevant information. But only when these unselected aspects of our world are both unexpected and important does IB have practical consequences, which is also the case in the field of team sports.

Following the expertise approach (Williams \& Ericsson, 2005; Williams \& Ford, 2008), or taking the literature on the orienting of attention (Nougier \& Rossi, 1999) into consideration, one could not necessarily have expected the presented results among experienced adult basketball players. When the results are compared with those for less experienced adults, it is evident that basketball novices - students who had taken a basic basketball class during their sport science course-were more likely to experience IB. This is an interesting finding, since it suggests that individuals are less likely to experience IB in their domain of expertise than are individuals with little experience in this domain. To our knowledge, this study is the first to address this question. We replicated the findings of previous studies showing that an attentiondemanding task can even disrupt performance in highly practiced activities, such as driving (Most \& Astur, 2007; Strayer et al., 2003), flying (Haines, 1991), or even simple walking (Hyman, Boss, Wise, McKenzie, \& Caggiano, in press). Beyond this, we provided evidence that participants with more practice in a particular domain were less likely to experience IB than were participants with hardly any practice in this domain.

This finding is in line with the argumentation of Wickens (1992) in the field of sport decision making, who already argued that skilled decision makers have three advantages over their less skilled counterparts. First, they are able to select the most relevant cue(s) from visual information on the basis of perceptual chunking and, therefore, process contextual information more effectively. Second, they have more extensive knowledge of situational probabilities stored in long-term memory and are better able to calibrate their decisions to current probabilities and risks. Third, they display a tighter coupling between cue recognition, hypothesis formation, and decision-making outcomes. This argumentation is in line with the mentioned perceptualcognitive adaptations, described by Williams and Ford (2008), that occur in expert team ball players. Nevertheless, it is remarkable that even adult basketball players, who have been training tactical decision making for a long time, seem to be distracted by a simple instruction directing their focus of attention and, for this reason, not include an open team member into their decision-making process.

Evidence from the attentional-orienting literature in the field of sport (for reviews, see Memmert, 2009; Nougier \& Rossi, 1999) suggests that expert athletes seem to pay proportionally less attention to highly likely events and more attention to less likely events, which Nougier and Rossi interpreted as adopting an expectingthe-unexpected strategy. The basketball players in the present study did not seem to adopt this strategy. A possible explanation for this might be that the attentiondemanding task of monitoring their direct opponent, combined with the hint that detecting the direct opponent as being either close to them or far away might aid them in determining their tactical decision, automatically triggered internalized if-then rules, such as the following: "If my direct opponent is far away from me, then I will shoot; or, if he is close to me, then I will do a quick first step and cut to the basket." Therefore, their attentional flexibility might have been reduced, not allowing them to utilize their expecting-the-unexpected strategy, which, according to Pesce et al. (2007), gives experienced athletes the advantage of rapidly zooming out visuospatial attention, allowing them to process unexpected but useful information. Experienced athletes might not adopt the expecting-the-unexpected strategy for every situation but, instead, adapt their strategy in accord with the game situation and zoom in visuospatial attention in predetermined offensive play strategies, whereas they might zoom out their visuospatial attention in open situations, in which the playmaker has not announced any specific strategy. Further research is needed to investigate this point in more depth.

Experiments 3 and 4 were able to significantly improve the ecological validity of the IB paradigm in the field of sport by improving perception-action coupling, which is highly important when attention processes are investigated in the field of sport (cf. Williams et al., 1999). Our results showed further that even in more realistic and, therefore, challenging decision-making situations with a more complex attention-demanding task, adult basketball players fail to perceive an obviously unguarded player as their optimal solution for this situation. Blackmore, Brelstaff, Nelson, and Troscianko (1995) argued that people in general believe that they see a complete, dynamic picture of a stable, uniformly detailed, and colorful world and are not aware of the fact that this stable visual world is constructed out of a brief retinal image and a very sketchy, higher level representation, along with a pop-out mechanism to redirect attention. For this reason, experiments of the kind described here surprise athletes, since they may miss an obviously unguarded team member when their attention is otherwise engaged, even though he appears to be right in the center of their visual field. 
Taken together, the present findings are highly relevant from a practical perspective. In most team sports, it is considered state of the art to practice precise predetermined offensive plays, especially in American football, basketball, handball, and so forth. These offensive routines reduce the complexity of the game by guiding the visual attention of the decision maker (e.g., the quarterback). The decision maker has to focus only on selected aspects of a specific constellation, because every player has received precise instructions on how to behave. Since the decision maker focuses his attention accordingly, he has only limited options, oftentimes in the form of ifthen rules, to choose from. Our findings indicate that this method is not always beneficial and athletes would oftentimes benefit from fewer instructions, leading to more creative behavior, which, in turn, would make the athlete less predictable for the opponent. Especially when training tactical decision making, it is beneficial to induce a broad breadth of attention by giving fewer instructions and not rigidly practicing offensive routines (Memmert, 2007).

However, care must be taken not to draw too strong inferences about the IB findings in the field of sport. As Simons (2007) mentioned for the psychology literature, IB tasks rely on a single critical trial to determine whether or not an unexpected object was consciously perceived; this inference depends on a report after the trial, and such reports are subject to many influences other than just whether or not the object was seen. For example, some people might be more hesitant to report something unlikely when they lack confidence that they saw it (in signal detection terms, they respond conservatively). This might be even more true in the presented decision-making setting in sport, since players might be biased by numerous factors, such as individual preferences based on their special skills (e.g., passing skills or confidence in their jump shot) or interindividual differences in their obedience to the instructions of the coach. However, this cannot explain the difference in the performance between the critical trial and the full-attention trial, since the only variation here was the presence of the attention-demanding task.

Nevertheless, one might still argue that the difference in performance between these two trials can be explained as a kind of practice effect, since the participants viewed the same video twice, first on the critical trial and then again on the full-attention trial. This explanation seems fairly unlikely, because the participants were not aware of the fact that they were seeing the same video again on the full-attention trial and, furthermore, did not seem to notice this afterward when they were asked whether they had noticed anything unusual while performing the task. Moreover, this explanation does not give any kind of information about why participants failed to detect the free teammate on the critical trial, which is the issue of concern in the reported studies. Furthermore, this is the standard and necessary procedure in the IB paradigm (Mack \& Rock, 1998; Most et al., 2005; Simons \& Chabris, 1999), because otherwise the unexpected object would not be unexpected. Another issue that is evident in all three experiments is the substantial number of participants ex- cluded. This can be explained by the fact that conducting research on situations that are unexpected is very challenging. Thus, almost all studies on IB have high rates of participants excluded, ranging from approximately $15 \%$ to almost 30\% (Mack \& Rock, 1998; Most et al., 2005; Simons \& Chabris, 1999).

In conclusion, these results need to be confirmed and further scrutinized. A first important step would be to show that the IB effect occurs generally and is not dependent on the position of the player. So far, IB has been shown only in very specific situations involving, for example, the perspective of backcourt players in handball and basketball. The question is whether IB can also be shown for different positions in basketball and handball or, for example, for the quarterback in American football or the goalkeeper in soccer or field hockey.

A second step must be to analyze the underlying attentional mechanisms athletes utilize while being involved in an IB task. Memmert (2006), for example, showed that observers who did not notice the unexpected object in the basketball test of Simons and Chabris (1999) spent, on average, as much time (about $1 \mathrm{sec}$ ) looking at the unexpected object as did those participants who did perceive it. Further eyetracking studies could focus on the different and more elaborate visual search strategies of team sport players. A first study by Laurent, Ward, Williams, and Ripoll (2005) indicated that experts made greater use of visual search strategies independently of local stimuli manipulations, as compared with novices, in a samedifferent judgment paradigm. It would be interesting to compare the search strategies of players who did perceive the open player with the strategies of those who missed him. This would, on the one hand, allow the identification of cognitively driven visual search strategies and would, on the other hand, reveal the organization of visual search in the presence of an unexpected object (in this case, an open player).

From a practical perspective, all the results of the different experiments presented have implications for the design of attention and decision-making training programs. Especially team sport players have to take in and process a great amount of information within a very short time in all sports games. They have to pay attention to sensory impressions that are often fairly unfamiliar to them and, because of that, are also often unexpected. This raises the question of how team players can become more proficient at perceiving constant minor and major changes in situations, caused by the interaction of their opponents and team members, if their attention has been directed to only a few specific aspects of the situation by the coach beforehand. Coaches are challenged to find ways of increasing their players' proficiency at identifying tactical solutions parallel to those they receive from the coaches, since it is not possible for the coach to mention all possible solutions for any situation or for the players to remember all of them. For example, when training specific offensive plays, coaches should mention that players nevertheless have to remain flexible and not rely entirely on the expected situation's occurring at the end of the play, since the defense might not act in the anticipated way, resulting in an unexpected open player. 


\section{AUTHOR NOTE}

Correspondence concerning this article should be addressed to P. Furley, Institute of Cognitive and Team/Racket Sport Research, German Sport University Cologne, Am Sportpark Müngersdorf 6, 50933 Köln, Germany (e-mail: p.furley@dshs-koeln.de).

\section{REFERENCES}

Abernethy, B. (2001). Attention. In R. N. Singer, H. A. Hausenblas, \& C. Janelle (Eds.), Handbook of research on sport psychology (pp. 5385). New York: Wiley.

Abernethy, B., MaXwell, J. P., Masters, R. S. W., VAN Der Kamp, J., \& JACKSON, R. C. (2007). Attentional processes in skill learning and expert performance. In G. Tenenbaum \& R. C. Eklund (Eds.), Handbook of sport psychology (3rd ed., pp. 245-263). Hoboken, NJ: Wiley.

Blackmore, S. J., Brelstaff, G., Nelson, K., \& Troscianko, T. (1995). Is the richness of our visual world an illusion? Transsaccadic memory for complex scenes. Perception, 24, 1075-1081.

Broadbent, D. E. (1958). Perception and communication. New York: Pergamon.

Brodeur, D. A., \& EnNS, J. T. (1997). Covert visual orienting across the lifespan. Canadian Journal of Experimental Psychology, 51, 20-35.

Castiello, U., \& Umiltà, C. (1992). Orienting of attention in volleyball players. International Journal of Sport Psychology, 23, 301-310.

Cereatti, L., Casella, R., Manganelli, M., \& Pesce, P. (2009). Visual attention in adolescents: Facilitating effects of sport expertise and acute physical exercise. Psychology of Sport \& Exercise, 10, 136-145.

Chun, M. M., \& Marois, R. (2002). The dark side of visual attention. Current Opinion in Neurobiology, 12, 184-189.

Davids, K., Button, C., Araújo, D., Renshaw, I., \& Hristovski, R. (2006). Movement models from sports provide representative task constraints for studying adaptive behavior in human movement systems. Adaptive Behavior, 14, 73-95.

EnNS, J. T., \& RichaRdS, J. C. (1997). Visual attentional orienting in developing hockey players. Journal of Experimental Child Psychology, 64, 255-275.

Ericsson, K. A., Krampe, R. T., \& Tesch-Römer, C. (1993). The role of deliberate practice in the acquisition of expert performance. Psychological Review, 100, 363-406.

Farrow, D., \& Abernethy, B. (2003). Do expertise and the degree of perception-action coupling affect natural anticipatory performance? Perception, 32, 1127-1139.

HAINES, R. F. (1991). A breakdown in simultaneous information processing. In G. Obrecht \& L. W. Stark (Eds.), Presbyopia research: From molecular biology to visual adaptation (pp. 171-175). New York: Plenum.

Hyman, I. E., JR., Boss, S. M., Wise, B. M., McKenzie, K. E., \& CaGGIANO, J. M. (in press). Did you see the unicycling clown? Inattentional blindness while walking and talking on a cell phone. Applied Cognitive Psychology.

KnUdSEn, E. I. (2007). Fundamental components of attention. Annual Review of Neuroscience, 30, 57-78.

Laurent, E., Ward, P., Williams, A. M., \& Ripoll, H. (2005). Expertise in basketball modifies perceptual discrimination abilities, underlying cognitive processes, and visual behaviours. Visual Cognition, $13,247-271$.

Lum, J., EnNs, J. T., \& Pratt, J. (2002). Visual orienting in college athletes: Explorations of athlete type and gender. Research Quarterly for Exercise \& Sport, 73, 156-167.

MACK, A., \& Rock, I. (1998). Inattentional blindness. Cambridge, MA: MIT Press.

Memmert, D. (2006). The effects of eye movements, age, and expertise on inattentional blindness. Consciousness \& Cognition, 15, 620-627.

Memmert, D. (2007). Can creativity be improved by an attentionbroadening training program? An exploratory study focusing on team sports. Creativity Research Journal, 19, 281-292.

Memmert, D. (2009). Pay attention! A review of attentional expertise in sport. International Review of Sport \& Exercise Psychology, 2, 119138.
Memmert, D., \& Furley, P. (2007). "I spy with my little eye!”-Breadth of attention, inattentional blindness, and tactical decision making in team sports. Journal of Sport \& Exercise Psychology, 29, 365-381.

Memmert, D., Simons, D., \& Grimme, T. (2009). The relationship between visual attention and expertise in sports. Psychology of Sport \& Exercise, 10, 146-151.

Most, S. B., \& Astur, R. S. (2007). Feature-based attentional set as a cause of traffic accidents. Visual Cognition, 15, 125-132.

Most, S. B., Scholl, B. J., Clifford, E. R., \& Simons, D. J. (2005). What you see is what you set: Sustained inattentional blindness and the capture of awareness. Psychological Review, 112, 217-242.

Nougier, V., Azemar, G., Stein, J.-F., \& Ripoll, H. (1992). Covert orienting to central visual cues and sport practice relations in the development of visual attention. Journal of Experimental Child Psychology, 54, 315-333.

Nougier, V., \& Rossi, B. (1999). The development of expertise in the orienting of attention. International Journal of Sport Psychology, 30, 246-260.

Pesce, C., Tessitore, A., Casella, R., Pirritano, M., \& Capranica, L. (2007). Focusing of visual attention at rest and during physical exercise in soccer players. Journal of Sports Sciences, 25, 1259-1270.

Pesce-Anzeneder, C., \& Bösel, R. (1998). Modulation of the spatial extent of the attentional focus in high-level volleyball players. European Journal of Cognitive Psychology, 10, 247-267.

PosNer, M. I. (1980). Orienting of attention. Quarterly Journal of Experimental Psychology, 32, 3-25.

Ranganathan, R., \& Carlton, L. G. (2007). Perception-action coupling and anticipatory performance in baseball batting. Journal of Motor Behavior, 39, 369-380.

Simons, D. J. (2007). Inattentional blindness. Scholarpedia, 2(5), 3244. Retrieved October 22, 2009, from www.scholarpedia.org/article/ Inattentional_blindness.

Simons, D. J., \& Chabris, C. F. (1999). Gorillas in our midst: Sustained inattentional blindness for dynamic events. Perception, 28, 1059-1074.

Strayer, D. L., Drews, F. A., \& Johnston, W. A. (2003). Cell phone induced failures of visual attention during simulated driving. Journal of Experimental Psychology: Applied, 9, 23-32.

Tenenbaum, G., \& Bar-Eli, M. (1995). Personality and intellectual capabilities in sport psychology. In D. H. Saklofske \& M. Zeidner (Eds.), International handbook of personality and intelligence (pp. 687-710). New York: Plenum.

Tenenbaum, G., Levy-Kolker, N., Sade, S., Liebermann, D. G., \& LIDOR, R. (1996). Anticipation and confidence of decisions related to skilled performance. International Journal of Sport Psychology, 27, 293-307.

Vaeyens, R., Lenoir, M., Williams, A. M., Mazyn, L., \& PhilipPAERTS, R. M. (2007). The effects of task constraints on visual search behavior and decision-making skill in youth soccer players. Journal of Sport \& Exercise Psychology, 29, 156-175.

Ward, P., \& Williams, A. M. (2003). Perceptual and cognitive skill development in soccer: The multidimensional nature of expert performance. Journal of Sport \& Exercise Psychology, 25, 93-111.

WICKENS, C. D. (1992). Engineering psychology and human performance. New York: HarperCollins.

Williams, A. M., Davids, K., \& Williams, J. G. (1999). Visual perception and action in sport. London: E \& FN Spon.

Williams, A. M., \& ERICsSON, K. A. (2005). Some considerations when applying the expert performance approach in sport. Human Movement Science, 24, 283-307.

Williams, A. M., \& Ford, P. R. (2008). Expertise and expert performance in sport. International Review of Sport \& Exercise Psychology, 1, 4-18.

Williams, A. M., Hodges, N. J., North, J. S., \& Barton, G. (2006). Perceiving patterns of play in dynamic sport tasks: Identifying the essential information underlying skilled performance. Perception, $\mathbf{3 5}$, 317-332.

(Manuscript received July 15, 2009 revision accepted for publication February 7, 2010.) 